\title{
Automata, Borel functions and real numbers in Pisot base
}

\author{
B. Cagnard ${ }^{\mathrm{a}}$, P. Simonnet ${ }^{\mathrm{a}}$, \\ ${ }^{\mathrm{a}}$ UMR CNRS 6134 Université de Corse BP 52 20250 CORTE, France
}

\begin{abstract}
This note is about functions $f: A^{\omega} \rightarrow B^{\omega}$ whose graph is recognized by a Büchi finite automaton on the product alphabet $A \times B$. These functions are Baire class 2 in the Baire hierarchy of Borel functions and it is decidable whether such fonction are continuous or not. In $1920 \mathrm{~W}$. Sierpinski showed that a function $f: \mathbb{R} \rightarrow \mathbb{R}$ is Baire class 1 if and only if both the overgraph and the undergraph of $f$ are $F_{\sigma}$. We show that such characterization is also true for functions on infinite words if we replace the real ordering by the lexicographical ordering on $B^{\omega}$. From this we deduce that it is decidable whether such fonction are of Baire class 1 or not. We extend this result to reals functions definable by automata in Pisot base.
\end{abstract}

Key words: Borel set, Borel function, automata, sequential machine.

\section{Introduction}

Very often in computer arithmetic a carry propagates from right to left. In $[5,16]$ on-line algorithms are proposed to compute arithmetic expressions from left to right. In general, on-the-fly algorithm process data in a serial manner from the most significant to the least significant digit. These algorithms however use several registers, each of them representing a correct prefix of the result, corresponding to an assumed value of the carry. In $[5,16]$ is presented a theoretical framework which allows to easily obtain on the fly algorithms whenever it is possible. C. Frougny [11] shows that a function is computable on the fly if and only if it is computable by a right subsequential finite state machine. The idea to read from left to right in a right subsequential finite state machine suggests non determinism. Moreover, working on infinite words

Email addresses: cagnard@univ-corse.fr (B. Cagnard), simonnet@univ-corse.fr (P. Simonnet). 
rather than finite words suggests discontinuity. A natural hierarchy exists on discontinuous Borel functions, the Baire classes of functions. The function $f$ belongs to class 0 if it is continuous. The function $f$ belongs to class 1 if it is the pointwiselimit of a sequence of functions of class 0 . The function $f$ belongs to class 2 if it is the pointwiselimit of a sequence of functions of class 1 , and so on. The present work concerns functions $f: A^{\omega} \rightarrow B^{\omega}$ whose graph is recognized by a Büchi finite automaton on the product alphabet $A \times B$ from a topological point of view. Topology and automata on infinite word have been heavily studied. It is easy to see that our functions are of Baire class 2, we prove that we can decide if they are of Baire class 1. We also prove this same result by interpreting these functions in a Pisot base manner. This extend the applicability of our result to the domain of real numbers. Our proof uses an old result of Sierpinski on Baire class 1 functions and decidability results of Landweber. The set of points of continuity of a function $f$ on an infinite word is always a countable intersection of open sets which is dense whenever $f$ is of Baire class 1. We expect that our approach will shed new light on the discussion in the field of on-the-fly algorithms. For this reason we present a detailled study of the Booth canonical recoding on infinite word. This function is an example of a discontinuous first class function.

The paper is organized as follows. First in sections 2, 3, 4 we present some necessary definitions and properties from automata theory and descriptive set theory. In section 5 we prove our decidability result on infinite words. In section 6 we study the Booth canonical recoding. In section 7 we prove our decidability result in the case where our functions define functions on real numbers in Pisot base. In the conclusion we advance our impressions on the asynchronuous case, that is to say the case of functions whose graph is recognized by Büchi automaton which transitions are labeled by couples of words $(u, v) \in A^{*} \times B^{*}$ instead of couples of letters $(a, b) \in A \times B$.

\section{Infinite words on a finite alphabet}

We note $\omega$ the set of natural numbers. Let $A$ be a finite alphabet and $<$ a linear order on $A$. All alphabets that we consider will have at least two letters. We denote $a$ the smallest element (first letter) of $A$ and $z$ the greatest element. A finite word $u$ on the alphabet $A$ is a finite sequence of elements of $A: u=u(0) u(1) \ldots u(n)$ where all the $u(i)$ 's are in $A$. The set of finite words on $A$ will be denoted $A^{*}$. The length (number of letters) of a word $u$ will be noted $|u|$. A particular word is the empty word $\epsilon,|\epsilon|=0$. The set $A^{+}$is $A^{*}-\{\epsilon\}$. With concatenation, $A^{*}$ is a monoid with unit element $\epsilon$. There is a natural order on $A^{*}$ : the lexicographical ordering, still denoted by $<$.

Lemma 2.1 Let $n$ be in $\omega$, we note $A^{n}$ the set of words $u \in A^{*}$ with $|u|=n$. 
(i) For all $n \in \omega-\{0\}$, every word $u \in A^{n}$ different of $a^{n}$ have an immediate predecessor in $A^{n}$ noted $\underline{u}$, for the lexicographical ordering.

(ii) For all $n \in \omega-\{0\}$, every word $u \in A^{n}$ different of $z^{n}$ have an immediate successor in $A^{n}$ noted $\bar{u}$ for the lexicographical ordering.

Proof: By induction on $n$ the length of $u$. If $u=v l$ with $v \in A^{n-1}$ and $l \in A$ then :

if $l \neq a$ or $z: \underline{u}=v(l-1)$ and $\bar{u}=v(l+1)$,

if $l=a: \underline{u}=\underline{v} z$ and $\bar{u}=v(a+1)$,

if $l=z: \underline{u}=v(z-1)$ and $\bar{u}=\bar{v} a$.

An infinite word $\alpha$ on the alphabet $A$ is an infinite sequence of elements of $A: \alpha=\alpha(0) \alpha(1) \ldots \alpha(n) \ldots$. The set of infinite words on the alphabet $A$ will be noted $A^{\omega}$. We note $\alpha[n]$ the finite word formed with the $n$ first letters of the infinite word $\alpha, \alpha[0]=\epsilon, \alpha[1]=\alpha(0)$. The set $A^{\omega}$, viewed as a product of infinitely many copies of $A$ with the discrete topology, is a metrizable space. It is equipped with the usual distance $d$ defined as follows. Let $\alpha, \beta \in A^{\omega}$,

$$
\begin{aligned}
& d(\alpha, \beta)=1 / 2^{n} \text { with } n=\min \{i \in \omega \mid \alpha(i) \neq \beta(i)\} \text { if } \alpha \neq \beta \\
& d(\alpha, \beta)=0 \text { if } \alpha=\beta
\end{aligned}
$$

The collection $\left(u A^{\omega}\right)_{u \in A^{*}}$ is a basis of clopen sets for this topology. Recall that $\left(A^{\omega}, d\right)$ is a compact metric space. The set $A^{\omega}$ is ordered by the lexicographical ordering $<$.

\section{Automata on infinite words}

For all this section, one can see [19].

Definition 3.1 A Büchi (nondeterministic) automaton $\mathcal{A}$ is a 5-tuple $: \mathcal{A}=<$ $A, Q, I, T, F>$, where $A$ is a finite alphabet, $Q$ is a finite set of states, $I \subset Q$ is the set of initial states, $T \subset Q \times A \times Q$ is the set of transitions and $F \subset Q$ the set of final states.

An infinite word $\alpha \in A^{\omega}$ is recognized by $\mathcal{A}$ if there is $\beta \in Q^{\omega}$ so that: $\beta(0) \in I, \forall n \in \omega,(\beta(n), \alpha(n), \beta(n+1)) \in T$ and there exists infinitely many $n$ 's so that $\beta(n) \in F$.

The set of words recognized by a Büchi automaton $\mathcal{A}$ is noted $L^{\omega}(\mathcal{A})$.

Let us denote by $\mathcal{P}(Q)$ the power set of $Q$. Notice that $T$ can be viewed as a partial function $\delta: Q \times A \rightarrow \mathcal{P}(Q)$ where $\delta(p, a)=\{q \in Q \mid(p, a, q) \in T\}$. By defining $\delta(p, u b)=\bigcup_{q \in \delta(p, u)} \delta(q, b)$ and $\delta(p, \epsilon)=\{p\}, \delta$ can be extended to a partial function $\delta: Q \times A^{*} \rightarrow \mathcal{P}(Q)$. 
A path $c$ of label $\alpha$ in $\mathcal{A}$ is an infinite word $c=c(0) c(1) \ldots c(n) \ldots \in(Q \times A \times Q)^{\omega}$ so that $\forall n \in \omega, c(n)$ is of the form $(\beta(n), \alpha(n), \beta(n+1))$ with $\beta(0) \in I$ and $c(n) \in T$.

$$
c=\beta_{0} \stackrel{\alpha_{0}}{\longrightarrow} \beta_{1} \stackrel{\alpha_{1}}{\longrightarrow} \beta_{2} \stackrel{\alpha_{2}}{\longrightarrow} \ldots
$$

Let us note Infinity $(c)$ the set of states which appears infinitely many times in $c$. A path $c$ of label $\alpha$ is successful if the word $\alpha$ is recognized by $\mathcal{A}$, it means that Infinity $(c) \cap T \neq \emptyset$.

An automaton is called deterministic if it has an unique initial state and for each state $p$ and each letter $a$ there exists at most one transition $(p, a, q) \in T$. In this case the partial transition function $\delta$ can be can be viewed as $\delta$ : $Q \times A \rightarrow Q$. For all infinite word $\alpha$ there exist, then, at most one path $c$ of label $\alpha$.

Example 1 Let $\mathcal{A}$ be the Büchi automaton on alphabet $A=\{0,1\} \times\{0,1\}$, with states $Q=\{1,2,3,4,5\}$, initial states $I=\{1,3,4\}$, final states $F=\{1,3,5\}$ and transitions

$$
\begin{aligned}
T=\{(1,(0,0), 1),(1,(1,1), 2),(2,(0,0), 1),(2,(1,1), 2) \\
(3,(1,1), 3),(4,(0,0), 4),(4,(1,1), 4),(4,(0,1), 5),(5,(1,0), 5)\}
\end{aligned}
$$

The graphical representation of $\mathcal{A}$ is given in Figure 1, the initial (resp. final) states are represented using an ingoing (resp. outgoing) unlabeled arrow . This automaton recognizes the graph of the function $S:\{0,1\}^{\omega} \rightarrow\{0,1\}^{\omega}$ defined by $S(\alpha)=\alpha$ if $\alpha$ has an infinite number of zeroes, $S\left(1^{\omega}\right)=1^{\omega}$ and for all $u \in\{0,1\}^{*} S\left(u 01^{\omega}\right)=u 10^{\omega}$. Let $\mu_{2}:\{0,1\}^{\omega} \longrightarrow[0,1]$ defined by $\mu_{2}(\alpha)=\sum_{i=0}^{\infty} \frac{\alpha(i)}{2^{i+1}}$. One can easily verify that for all $\alpha \in\{0,1\}^{\omega}, S(\alpha)$ is the maximum lexicographic of the binary representations of $\mu_{2}(\alpha)$. S is known as normalization in base 2.

Definition 3.2 A Muller automaton $\mathcal{A}$ is a 5-tuple : $\mathcal{A}=\langle A, Q, I, T, \mathcal{F}>$, where $A$ is a finite alphabet, $Q$ is a finite set of states, $I \subset Q$ is the set of initial states, $T \subset Q \times A \times Q$ is the set of transitions and $\mathcal{F} \subset \mathcal{P}(Q)$. The difference between Büchi automata and Muller automata is the acceptance condition.

An infinite word $\alpha \in A^{\omega}$ is recognized by $\mathcal{A}$ if there is an infinite path $c$ of label $\alpha$ so that Infinity $(c) \in \mathcal{F}$. A deterministic Muller automaton is defined as in the case of deterministic Büchi automata.

Consider the following logical language : the set $\mathcal{V}$ of the variables, its elements noted by $x, y, z \ldots$, a constant symbol 0 and a unary fonction $s$ (as successor). We define the set of the terms $\mathcal{T}$ by :

i) A variable is a term.

ii) 0 is a term.

iii) if $t \in \mathcal{T}$ then $s(t) \in \mathcal{T}$. 

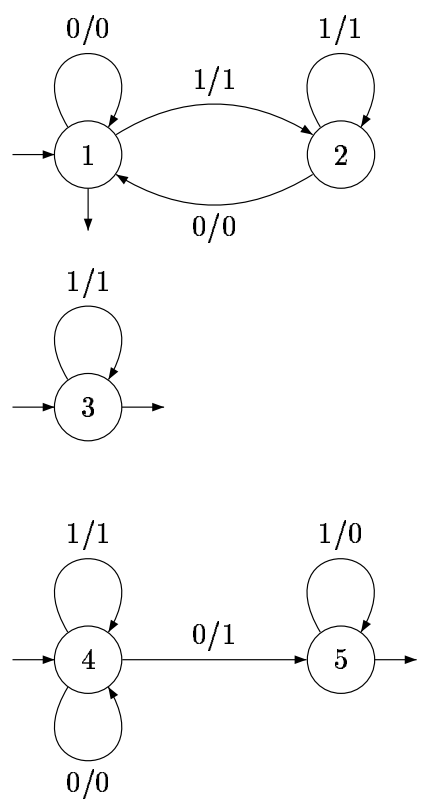

Figure 1. Normalization in base 2

Let $\mathcal{P}$ (as parts) another set of variables, this variables are noted $\mathcal{X}, \mathcal{Y}, \mathcal{Z} \ldots$ and two binary predicates $=, \in$. The atomic formulae are of the form $t=t^{\prime}$ with $\left(t, t^{\prime}\right) \in \mathcal{T}^{2}$ or $t \in \mathcal{X}$ with $t \in \mathcal{T}$ and $\mathcal{X} \in \mathcal{P}$.

Definition 3.3 A formula of $S 1 S$ is defined as following:

i) An atomic formula is in $S 1 S$.

ii) If $\phi \in S 1 S$ then $\neg \phi, \forall x \phi, \exists x \phi, \forall \mathcal{X} \phi, \exists \mathcal{X} \phi$ are in $S 1 S$, with $x \in \mathcal{V}$, $\mathcal{X} \in \mathcal{P}$

iii) If $\phi$ and $\psi$ are in $S 1 S$ then $\phi \wedge \psi, \phi \vee \psi, \phi \Rightarrow \psi, \phi \Leftrightarrow \psi$ are in $S 1 S$.

The interpretation of these formulae is the following : the variables of $\mathcal{V}$ are interpreted as natural numbers, the symbol 0 as $0 \in \omega$, the symbol $s$ as the successor function in $\omega$, the variables of $\mathcal{P}$ as subsets of $\omega$ and the predicates symbols as $=$ and $\in$ in $\omega$. If each integer is assimilated to a singleton and each subset of $\omega$ to an infinite word on the $\{0,1\}$ alphabet, then a $S 1 S$ formula $\phi\left(\mathcal{X}_{1}, \mathcal{X}_{2}, \ldots, \mathcal{X}_{n}\right)$, with $\mathcal{X}_{1}, \mathcal{X}_{2}, \ldots, \mathcal{X}_{n}$ free variables defines the $\omega$-language $L_{\phi} \subset$ $\underbrace{2^{\mathbb{N}} \times \ldots 2^{\mathbb{N}}}_{n}$ of the $n$-tuple of characteristic words satisfying $\phi$.

An $\omega$-language $L$ is said definable in $S 1 S$ if there exists a formula $\phi$ in $S 1 S$ so that $L=L_{\phi}$.

Recall the following result :

Theorem 3.4 for all $\omega$-language L, the following assertions are equivalent :

i) $L=\bigcup_{1 \leq i \leq n} A_{i} B_{i}^{\omega}$ with $A_{i}, B_{i}$ rational sets of finite words. 
ii) $L=L^{\omega}(\mathcal{A})$ with $A$ nondeterministic Büchi automaton.

iii) $L=L^{\omega}(\mathcal{A})$ with $A$ deterministic Muller automaton.

iv) $L$ is definable in $S 1 S$.

We call $\operatorname{Rec}\left(A^{\omega}\right)$ the family of such languages.

\section{Borel hierarchy}

For all this section, one can see $[15,19]$. Borel sets of a topological space $X$ are the sets obtained from open sets using complementation and countable unions. When $X$ is metrizable we can define the hierarchy of Borel sets of finite rank :

Definition 4.1 Let $X$ be a metrizable space, for $n \in \omega-\{0\}$, we define by induction the classes $\boldsymbol{\Sigma}_{n}^{0}(X), \boldsymbol{\Pi}_{n}^{0}(X)$ and $\boldsymbol{\Delta}_{n}^{0}(X)$ :

$\boldsymbol{\Sigma}_{1}^{0}(X)=G(X)$ the class of open sets of $X$

$\boldsymbol{\Pi}_{n}^{0}(X)=\left\{A^{\vee} \mid A \in \Sigma_{n}^{0}(X)\right\}$, where $A^{\vee}$ refers to the complement of $A$.

$\boldsymbol{\Sigma}_{n+1}^{0}(X)=\left\{\cup_{m} A_{m} \mid A_{m} \in \Pi_{n}^{0}(X), m \in \omega\right\}$

$\Delta_{n}^{0}(X)=\Sigma_{n}^{0}(X) \cap \Pi_{n}^{0}(X)$

In particular, we have :

$\Pi_{1}^{0}$ is the class of closed sets.

$\Sigma_{2}^{0}=F_{\sigma}$ is the class of countable unions of closed sets.

$\boldsymbol{\Pi}_{2}^{0}=G_{\delta}$ is the class of countable intersections of open sets.

One can prove that : $\boldsymbol{\Sigma}_{n}^{0} \cup \boldsymbol{\Pi}_{n}^{0} \subset \boldsymbol{\Delta}_{n+1}^{0}$.

This gives us the following picture where any class is contained in every class to the right of it :

$$
\begin{aligned}
& \Sigma_{1}^{0} \quad \Sigma_{2}^{0} \quad \Sigma_{3}^{0} \quad \Sigma_{n}^{0} \\
& \begin{array}{llllll}
\Delta_{1}^{0} & \Delta_{2}^{0} & \Delta_{3}^{0} & \ldots \Delta_{n} & \ldots
\end{array} \\
& \Pi_{1}^{0} \quad \Pi_{2}^{0} \quad \Pi_{3}^{0} \quad \Pi_{n}^{0}
\end{aligned}
$$

The Borel hierarchy is also defined for transfinite levels [15], but we shall not need them in the present study.

For all $n \in \omega$ the classes $\boldsymbol{\Sigma}_{n}^{0}(X), \boldsymbol{\Pi}_{n}^{0}(X), \boldsymbol{\Delta}_{n}^{0}(X)$ are closed by finite union and intersection, moreover $\boldsymbol{\Sigma}_{n}^{0}(X)$ is closed by countable union, $\boldsymbol{\Pi}_{n}^{0}(X)$ closed by countable intersection and $\boldsymbol{\Delta}_{n}^{0}(X)$ closed by complement.

When $X$ is an uncountable metric complete space, the Borel hierarchy is strict. In what follows $X$ will be $A^{\omega}$ or $[a, b]$ with $a$ and $b$ real numbers. 
Definition 4.2 The definition of Baire classes for functions is recursive. Let $X, Y$ be metrizable spaces and a function $f: X \rightarrow Y$.

i) $f$ is Baire class 0 if $f$ is continuous.

ii) $\forall n \in \omega, f$ is Baire class $(n+1)$ if $f$ is the pointwise limit of a sequence of Baire class $n$ functions.

The Lebesgue, Hausdorff, Banach theorem make the connexion with the Borel hierarchy, see [15] :

Theorem 4.3 Let $X, Y$ be metrizable spaces with $Y$ separable. Then for all $n \geq 2, f: X \rightarrow Y$ is Baire class $n$ iff for all open $V \in Y, f^{-1}(V) \in \Sigma_{n+1}^{0}(X)$.

Remark 1 Note that this result hold for $n=1$ if in addition $X$ is separable and either $X=A^{\omega}$ or else $Y=\mathbb{R}$.

Denote $\operatorname{cont}(f)$ the set of points of continuity of $f$. We have the classical following proposition, see [15] :

Proposition 4.4 Let $X, Y$, be metrizable spaces and $f: X \rightarrow Y$, then cont $(f)$ is $G_{\delta}$.

The following result due to Baire shows that Baire class 1 functions have many continuity points, see $[15]$ :

Theorem 4.5 Let $X, Y$, be metrizable spaces with $Y$ separable and $f: X \rightarrow$ $Y$ be Baire class 1 . Then cont $(f)$ is a dense $G_{\delta}$ set.

It is well known that the graph of a continuous functions is closed. The following result is classical, see [8] for example.

Lemma 4.6 Let $X, Y$, be metrizable spaces with $Y$ compact and $f: X \rightarrow Y$. $f$ is continuous iff its graph is closed.

Lemma 4.7 Let $X, Y$, be metrizable spaces with $Y$ separable and $f: X \rightarrow Y$. If $f$ is Baire class $n$ then its graph is $\mathbf{\Pi}_{n+1}^{0}(X)$.

Proof: We give the proof in the case $X=A^{\omega}, Y=B^{\omega}$. First notice that if $f(\alpha)=\beta$ then $\forall u \in B^{*},\left(\beta \in u B^{\omega} \Rightarrow f(\alpha) \in u B^{\omega}\right)$ and if $f(\alpha) \neq \beta$ then $\exists u \in B^{*}$ such that $\beta \in u B^{\omega}$ and $f(\alpha) \notin u B^{\omega}$. Thus :

$$
(\alpha, \beta) \in \operatorname{graph}(f) \Leftrightarrow f(\alpha)=\beta \Leftrightarrow\left[\forall u \in Y^{*}\left(\beta \in u B^{\omega} \Rightarrow f(\alpha) \in u B^{\omega}\right)\right]
$$

As $f$ is Baire class $n,\left\{\alpha \in A^{\omega} \mid f(\alpha) \in u B^{\omega}\right\}$ is in $\Delta_{n+1}^{0}\left(A^{\omega}\right)$ and $\left\{\beta \in B^{\omega} \mid \beta \in u B^{\omega}\right\}$ is in $\Delta_{1}^{0}\left(B^{\omega}\right)$. Thus for all fixed $u \in B^{*}$, $\left\{(\alpha, \beta) \in A^{\omega} \times B^{\omega} \mid\left(\beta \in u B^{\omega} \Rightarrow f(\alpha) \in u B^{\omega}\right)\right\}$ is in $\Delta_{n+1}^{0}\left(A^{\omega} \times B^{\omega}\right)$ and $\left\{(\alpha, \beta) \in A^{\omega} \times B^{\omega} \mid \forall u \in B^{*}\left(\beta \in u B^{\omega} \Rightarrow f(\alpha) \in u B^{\omega}\right)\right\}$ is in $\Pi_{n+1}^{0}\left(A^{\omega} \times B^{\omega}\right)$. 


\section{We can decide if a function definable in $S 1 S$ is Baire class 1}

Definition 5.1 Let $A, B$ be finite alphabets a function $f: A^{\omega} \rightarrow B^{\omega}$ is definable in $S 1 S$ if its graph is defined by a formula in $S 1 S$.

Thanks to Theorem $3.4 f: A^{\omega} \rightarrow B^{\omega}$ is definable in $S 1 S$ if its graph is recognized by a Büchi automaton on the product alphabet $A \times B$.

Recall that $f$ is Baire class $n$ if $f^{-1}(U) \in \Sigma_{n+1}^{0}$ for every open set $U \in B^{\omega}$. As $\left(u B^{\omega}\right)_{u \in B^{*}}$ is a basis of clopen sets, this condition is equivalent to :

$$
\forall u \in B^{*}, \quad f^{-1}\left(u B^{\omega}\right) \in \Delta_{n+1}^{0}
$$

It is easy to see that sets recognizable by Muller automata are $\boldsymbol{\Delta}_{3}^{0}$, in fact they are boolean combination of $\Sigma_{2}^{0}$.

Proposition 5.2 Let $A, B$ be finite alphabets and $f: A^{\omega} \rightarrow B^{\omega}$ be a function definable in $S 1 S$. Then $f$ is Baire class 2.

Proof: We need only to remark that if $U$ is recognizable by a Muller automaton then $f^{-1}(U)$ is recognizable.

At last, let us recall a result of Landweber [14] :

Proposition 5.3 If $L \in \operatorname{Rec}\left(A^{\omega}\right)$ and $\Pi_{2}^{0}$ then $L$ is recognizable by a deterministic Büchi automaton.

Moreover one can decide for $L \in \operatorname{Rec}\left(A^{\omega}\right)$ if it is $\boldsymbol{\Sigma}_{i}^{0}$ (resp. $\left.\boldsymbol{\Pi}_{i}^{0}\right)$ for $i=1$, 2.

Let $f$ be definable in $S 1 S$, it is easy to see that cont $(f)$ is still definable in $S 1 S$. So by propositions 4.6 and $5.3 \operatorname{cont}(f)$ is recognizable by a deterministic Büchi automaton. Moreover if it is Baire class 1 then by proposition 4.7 its graph is recognizable by a deterministic Büchi automaton.

Definition 5.4 Let $f: A^{\omega} \rightarrow B^{\omega}$ be a function where $B^{\omega}$ is lexicographically ordered. The overgraph and the undergraph of $f$ are respectively:

$$
\begin{aligned}
& G \uparrow(f)=\left\{(\alpha, \beta) \in A^{\omega} \times B^{\omega} \mid f(\alpha)<\beta\right\} \\
& G \downarrow(f)=\left\{(\alpha, \beta) \in A^{\omega} \times B^{\omega} \mid f(\alpha)>\beta\right\}
\end{aligned}
$$


W. Sierpinski [24] has shown that a function $f: \mathbb{R} \rightarrow \mathbb{R}$ is Baire class 1 if and only if the overgraph and the undergraph of $f$ are $F_{\sigma}$. We show that this characterization is also true for functions on infinite words if we replace the real ordering by the lexicographical ordering on $B^{\omega}$.

Proposition 5.5 Let $A$ and $B$ be two finite alphabets, then $f: A^{\omega} \rightarrow B^{\omega}$ is Baire class 1 iff the overgraph and the undergraph of $f$ are in $F_{\sigma}$.

\section{Proof:}

$(\Rightarrow)$

Let $(\alpha, \beta) \in A^{\omega} \times B^{\omega}$. The word $f(\alpha)$ is lexicographically less than $\beta$ iff there exists $n \in \omega$ such that $f(\alpha)[n]=\beta[n]$, i.e., they have the same prefix of length $n$, and $f(\alpha)(n)<\beta(n)$. Let $u=f(\alpha)[n+1] \in B^{+}$, then $f(\alpha) \in u B^{\omega}$. So :

$$
G \uparrow(f)=\bigcup_{u \in B^{+}}\left(f^{-1}\left(u B^{\omega}\right) \times \bigcup_{v>u,|v|=|u|} v B^{\omega}\right)
$$

As $F_{\sigma}$ is closed by countable unions then the overgraph of $f$ is $F_{\sigma}$.

$(\Leftarrow)$

Let $u \in B^{+}$, we denote by $a$ the minimum and $z$ the maximum of $B$.

We first consider the case where $u$ is not of the form $a^{n}$ or $z^{n}$. We have :

$$
\begin{gathered}
\beta \in u B^{\omega} \Leftrightarrow \beta>\underline{u} z^{\omega} \text { and } \beta<\bar{u} a^{\omega} \\
\alpha \in f^{-1}\left(u B^{\omega}\right) \Leftrightarrow f(\alpha)>\underline{u} z^{\omega} \text { and } f(\alpha)<\bar{u} a^{\omega}
\end{gathered}
$$

Then $f^{-1}\left(u B^{\omega}\right)=\left\{\alpha \in B^{\omega} \mid f(\alpha)>\underline{u} z^{\omega}\right\} \bigcap\left\{\alpha \in B^{\omega} \mid f(\alpha)<\bar{u} a^{\omega}\right\}$

But $\left\{\alpha \in B^{\omega} ; f(\alpha)>\underline{u} z^{\omega}\right\}$ (respectively $\left\{\alpha \in B^{\omega} \mid f(\alpha)<\bar{u} a^{\omega}\right\}$ ) is $F_{\sigma}$ as section of the undergraph (respectively overgraph) of $f$ and this proves the result.

In the case where $u=a^{n}$, the proof is the same with $f^{-1}\left(u B^{\omega}\right)=\{\alpha \in$ $\left.B^{\omega} \mid f(\alpha)<\bar{u} a^{\omega}\right\}$. And for $u=z^{n}, f^{-1}\left(u B^{\omega}\right)=\left\{\alpha \in B^{\omega} \mid f(\alpha)>\underline{u} z^{\omega}\right\}$

Remark 2 Note that the notion of Baire class 1 is purely topological so it is independant of the order on $B$. So to be $F_{\sigma}$ for the overgraph and the undergraph is independent of the choice of the order on $B$.

Theorem 5.6 We can decide if a function $f: A^{\omega} \rightarrow B^{\omega}$ so that $\operatorname{Graph}(f)=\left\{(\alpha, \beta) \in A^{\omega} \times B^{\omega} \mid f(\alpha)=\beta\right\}$ is definable in $S 1 S$ is Baire class 1.

Proof: Fix an order on $B$. The lexicographical ordering on $B^{\omega}$ is definable in $S 1 S$. We have:

$$
(\alpha, \beta) \in G \downarrow(f) \Leftrightarrow \exists \gamma \in B^{\omega}((\alpha, \gamma) \in G \operatorname{Graph}(f) \wedge \beta<\gamma)
$$


Then the overgraph and the undergraph of $f$ are definable in $S 1 S$. Using proposition 5.3, we can decide if $f$ is Baire class 1 .

\section{An example of non continuous Baire class 1 function : the canon- ical Booth function}

In [11], C. Frougny shows that a function can be computed on-the-fly iff it is a right subsequential function. She gives as example the Booth canonical recoding, see also [18] for applications to multiplication. In this section, we extend the Booth canonical recoding on infinite words, prove that it is a non continuous Baire class 1 function and give its set of continuity points.

We recall the definition of a right subsequential function.

Definition 6.1 A right subsequential machine with input alphabet $A$ and output alphabet $B, \mathcal{M}=\left(Q, A \times B^{*}, T, i, s\right)$ is a directed graph labeled by elements of $A \times B^{*}$ where $Q$ is the set of states, $i \in Q$ is the initial state, $T \in Q \times\left(A \times B^{*}\right) \times Q$ is the set of labeled transitions and $s: Q \rightarrow B^{*}$ is the terminal function. The machine must satisfy the following property : it is input deterministic, i.e., if $p \stackrel{a / u}{\longrightarrow} q$ and $p \stackrel{a / v}{\longrightarrow} r$, then $q=r$ and $u=v$. A word $u=a_{0} \ldots a_{n} \in A^{*}$ has $v \in B^{*}$ for image by $\mathcal{M}$ if there exists a path in $\mathcal{M}$ starting in the initial state $i$

$$
i \stackrel{a_{n} / v_{n}}{\longrightarrow} q_{1} \stackrel{a_{n-1} / v_{n-1}}{\longrightarrow} \ldots q_{n} \stackrel{a_{0} / v_{0}}{\longrightarrow} q_{n+1}
$$

with $v_{i} \in B^{*}$ and such that $v=s\left(q_{n+1}\right) v_{0} \ldots v_{n}$.

A function $f: A^{*} \rightarrow B^{*}$ is right subsequential if there exists a right subsequential machine $\mathcal{M}$ such that if $u \in A^{*}$ and $v \in B^{*}, v=f(u)$ iff $v$ is the image of $u$ by $\mathcal{M}$.

On finite words, the Booth canonical recoding is the function that maps any binary representation onto an equivalent Aviezinis one with the minimum number of non-zero digits : $\varphi:\{0,1\}^{*} \rightarrow A^{*}$ with $A=\{\overline{1}, 0,1\}$ where $\overline{1}$ means -1 [18]. It can be obtained by a least significant digit first (LSDF) algorithm by replacing each block of the form $01^{n}$, with $n \geq 2$, by $10^{n-1} \overline{1}$. The following right subsequential machine realizes the Booth canonical recoding [11].

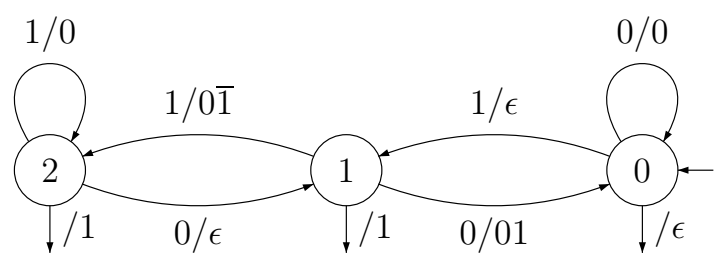

Figure 2. Right subsequential Booth canonical recoding 
We will now extend the Booth canonical recoding on infinite words $\alpha$ which $\alpha(0)=0, \varphi: 0\{0,1\}^{\omega} \rightarrow A^{\omega}$. First note that on finite words, the pattern 00 in the input blocks a possible carry. So for $\alpha \in 0\{0,1\}^{\omega}$ if $\alpha$ contains an infinity of 00 it is natural to extend Booth canonical recoding on $\alpha$ using the algorithm on each finite consecutive word of $\alpha$ starting by 00 .

Example 2 An infinite number of 00.

$$
\begin{array}{llll}
\varphi(01100101100010100011100 \ldots) & =\varphi(011) & \varphi(001011) & \varphi(000111) \ldots \\
& =10 \overline{1} & 010 \overline{1} 0 \overline{1} & 00100 \overline{1} \ldots \\
\varphi\left(0101(01010011011)^{\omega}\right) & =\varphi(0101) & (\varphi(0101) & \varphi(0011011))^{\omega} \\
& =0101 & (0101 & 0100 \overline{1} 0 \overline{1})^{\omega}
\end{array}
$$

If the number of 00 in $\alpha$ is finite we must be careful because a carry can come from the infinity. This case depends of the number of 11 contained in $\alpha$. If this number is finite : let $n$ be the greatest integer such that $\alpha(n-$ $2) \alpha(n-1)=11(n=0$ if no 11 hold in $\alpha)$ then we can extend $\varphi$ on $\alpha$ by $\varphi(\alpha)=\varphi(\alpha[n]) \alpha(n) \alpha(n+1) \ldots$

Example 3 A finite number of 00 and finite number of 11.

$\varphi\left((01)^{\omega}\right)=(01)^{\omega}$

$\varphi\left(01001011(0101001)^{\omega}\right)=01010 \overline{1} 0 \overline{1}(0101001)^{\omega}$

At last, if in $\alpha$ the number of 00 is finite and the number 11 is infinite then a carry come from the infinity and propagate up to the last 00 . Let then $n$ be the greatest integer such that $\alpha(n-1) \alpha(n)=00(n=0$ if no 00 hold in $\alpha)$. Therefore we can extend $\varphi$ on $\alpha$ by $\varphi(\alpha)=\varphi(\alpha[n]) 1 \psi(\alpha(n+1) \alpha(n+2) \ldots)$ with $\psi:\{0,1\}^{\omega} \rightarrow A^{\omega}$ the sequential function defined by $\psi(0)=\overline{1}$ and $\psi(1)=0$.

Example 4 A finite number of 00 and infinite number of 11.

$\varphi\left(01^{\omega}\right)=10^{\omega}$

$\varphi\left(01100(101011)^{\omega}\right)=10 \overline{1} 01(0 \overline{1} 0 \overline{1} 00)^{\omega}$

With this construction, we obtain a function $\varphi: 0\{0,1\}^{\omega} \rightarrow A^{\omega}$ which still maps any binary representation onto an equivalent Aviezinis one with the minimum number of non-zero digits.

The graph of $\varphi$ is realized by the Büchi automaton $\mathcal{A}$ of figure 3 .

The essential difference with the finite case is that the carry can come from the infinity and this suggest discontinuity. A block of the form 11 launch or propagate the carry and a block the form 00 stop the carry. So have finite or infinite number of such blocks will be important in the study of the regularity of $\varphi$. 


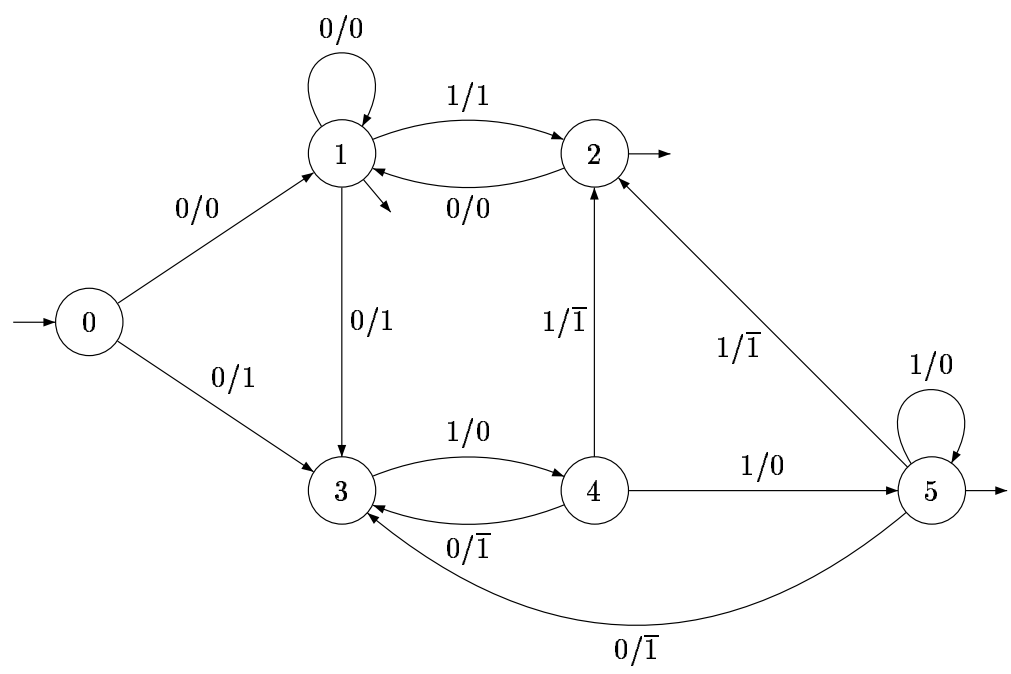

Figure 3. Booth Büchi automata

Proposition $6.2 \varphi: 0\{0,1\}^{\omega} \rightarrow A^{\omega}$ is a non continuous Baire class 1 function.

Proof: It is easy to see $\varphi$ as the pointwise limit of a sequence of continuous function but it is more interesting to determine the topological complexity of $\varphi^{-1}(V)$ for $V \in\left\{v A^{\omega} \mid v \in A^{*}\right\}$ basis of clopen sets of $A^{\omega}$.

(1) Let $\alpha \in \varphi^{-1}\left(v \overline{1} A^{\omega}\right)$ with $|v|=n$. It means that $\delta(0,(\alpha[n], v)) \neq \emptyset$ and $\bigcup_{q \in \delta(0,(\alpha[n], v))} \delta(q,(\alpha(n), \overline{1})) \neq \emptyset$. So there is two possibilities for $\alpha(n)$ : $\alpha(n)$ is a 0 which propagates a carry (transition from the state 4 to 3 or 5 to 3 ) or $\alpha(n)$ is a 1 which releases a carry (transition from the state 4 to 3 or 5 to 3$)$. Let $I=\left\{u \in\{0,1\}^{*}|| u \mid=n, \delta(0,(u, v)) \bigcap\{4,5\} \neq \emptyset\right\}, I$ is finite, and :

$$
\varphi^{-1}\left(v \overline{1} A^{\omega}\right)=\bigcup_{u \in I}\left(u 0(10)^{*} 11\{0,1\}^{\omega} \bigcup u 1(01)^{*} 00\{0,1\}^{\omega}\right)
$$

So $\varphi^{-1}\left(v \overline{1} A^{\omega}\right)$ is open.

(2) Let $\alpha \in \varphi^{-1}\left(v 0 A^{\omega}\right)$ with $|v|=n$. The two possibilities for $\alpha(n)$ are $: \alpha(n)$ is a 0 which does not propagate a carry (transition from the state 1 to 1 or 2 to 1 ) or $\alpha(n)$ is a 1 which propagate a carry (transition from the state 3 to 4,4 to 5 or 5 to 5$)$. Let $J=\left\{u \in\{0,1\}^{*}|| u \mid=n, \delta(0,(u, v)) \cap\{1,2\} \neq\right.$ $\emptyset\}, K=\left\{u \in\{0,1\}^{*}|\quad| u \mid=n, \delta(0,(u, v)) \cap\{3,4,5\} \neq \emptyset\right\}, J$ and $K$ are finite, and :

$$
\varphi^{-1}\left(v 0 A^{\omega}\right)=\bigcup_{u \in J}\left(u 0(10)^{*} 0\{0,1\}^{\omega} \bigcup u 0(10)^{\omega}\right) \bigcup_{u \in K} u 1(01)^{*} 1\{0,1\}^{\omega}
$$


So $\varphi^{-1}\left(v 0 A^{\omega}\right)$ is a closed non open set so $\varphi$ is not continuous.

(3) Let $\alpha \in \varphi^{-1}\left(v 1 A^{\omega}\right)$ with $|v|=n$. The two possibilities for $\alpha(n)$ are $: \alpha(n)$ is a 0 which stops a carry (transition from the state 1 to 3 ) or $\alpha(n)$ is a 1 which does not propagate a carry (transition from the state 1 to 2 ). Let $L=\left\{u \in\{0,1\}^{*}|| u \mid=n, \delta(0,(u, v))=\{1\}\right\}, I$ is finite, and :

$$
\varphi^{-1}\left(v 1 A^{\omega}\right)=\bigcup_{u \in L}\left(u 0(10)^{*} 11\{0,1\}^{\omega} \bigcup u 1(01)^{*} 00\{0,1\}^{\omega} \bigcup u 1(01)^{\omega}\right)
$$

So $\varphi^{-1}\left(v \overline{1} A^{\omega}\right)$ is a closed non open set.

Then for all open set $V \in A^{\omega}, \varphi^{-1}(V)$ is $F_{\sigma}$ and $\varphi$ is Baire class 1 .

Consider now the continuity points of $\varphi$. It is easy to see that it is not continuous in $(01)^{\omega}: \varphi\left((01)^{\omega}\right)=(01)^{\omega},(01)^{n} 1^{\omega}$ converges to $(01)^{\omega}$ and $\varphi\left((01)^{n} 1^{\omega}\right)=$ $1(0 \overline{1})^{n-1} 0^{\omega}$.

Proposition 6.3 The set of points of non continuity of $\varphi$ is $\left\{u(01)^{\omega} \mid u \in\right.$ $\left.0\{0,1\}^{*}\right\}$.

Proof: The function $\varphi$ is not continuous in $\alpha$ iff there exist an open set $V \in A^{\omega}$ so that $\alpha \in \varphi^{-1}(V) \backslash \operatorname{Int}\left(\varphi^{-1}(V)\right)$. In the proof of the precedent result, we have the complete description of $\varphi^{-1}(V)$ for a basis of open sets and such $\alpha$ are the words of the form $u(01)^{\omega}$.

Remark 3 Note that $\operatorname{cont}(\varphi)$ is quite a dense $G_{\delta}$ set.

Another example of function definable in $S 1 S$, Baire class 1 but not continuous, is given by the normalization (example 1) in Pisot numeration systems $[12]$.

\section{The case of the real numbers}

In this section we consider a numeration system for real numbers with Pisot base, i.e, $\theta$ is a Pisot number (like the golden mean $\frac{1+\sqrt{5}}{2}$ ) , $A \subset \mathbb{Z} \cap[0, \theta]$ and the function $\mu_{\theta}$ defined by :

$$
\begin{aligned}
\mu_{\theta}: A^{\omega} & \rightarrow[0,1] \\
\alpha & \mapsto \Sigma_{n \geq 0} \frac{\alpha(n)}{\theta^{n+1}}
\end{aligned}
$$

Let us recall that $\mu_{\theta}$ is a continuous surjection on $[0,1]$.

C. Frougny proved that $M=\left\{(\alpha, \beta) \in A^{\omega} \times A^{\omega} \mid \mu_{\theta}(\alpha)=\mu_{\theta}(\beta)\right\}$ and $N=\left\{(\alpha, \beta) \in A^{\omega} \times A^{\omega} \mid \mu_{\theta}(\alpha)<\mu_{\theta}(\beta)\right\}$ are definable in $S 1 S$ [10] see also [2]. 
From now on, we consider functions $f: A^{\omega} \rightarrow A^{\omega}$ definable in $S 1 S$ which are consistent with $\mu_{\theta}$, i.e., functions $f$ definable in $S 1 S$ such that there exists $F$ such that the following diagram commutes:

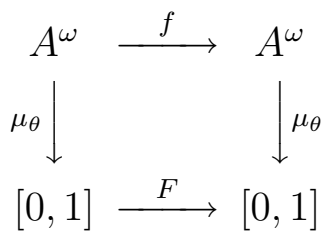

In the case of base $k$, one can find historical examples of such continuous $F$ in the chapter XIII of S.Eilenberg [4]. In 1890 Giuseppe Peano published an example of a continuous function

$$
H:[0,1] \longrightarrow[0,1] \times[0,1]
$$

which is surjective, the square-filling curve. We have $H=(F, G)$ with $F:[0,1] \longrightarrow[0,1], G:[0,1] \longrightarrow[0,1]$. The function $F$ (resp $G$ ) can be defined by a consistent function $f: 9^{\omega} \longrightarrow 3^{\omega}$ from base 9 to base 3 . The function $f$ is realized by a left sequential letter to letter transducer, hence $f$ is definable in $S 1 S$. Other examples in the same spirit can be find in the works of Waclaw Sierpinski, Bernard Bolzano, Ludwig Scheeffer, Georg Cantor. The reader interested in history should see the book of A. Edgar [3] and the beautiful article of B. Maurey and J.P. Tacchi [17] about the devil's staircase of Ludwig Scheeffer. One can describe non continuous examples with $f$ definable in $S 1 S$ and Pisot Basis. For example some historical functions of the analysis like jumps function that we have seen in [17]. For simplify we suppose that we have the same Pisot number in input and output. Note that if $f$ is definable in $S 1 S$, and if $\theta$ is a Pisot number then one can decide if $f$ is consistent. This can be expressed by a closed $S 1 S$ formula and $S 1 S$ is decidable [1]. For more details, we refer the reader to [2].

As $f$ is Baire class 2, the topological complexity of such $F$ is Baire class 2. To see this we can use the following theorem of Saint Raymond [15,22].

Theorem 7.1 Let $X, Y$ be compact metrizable spaces, $Z$ a separable metrizable space, a continuous surjection $g: X \rightarrow Y$ and a Baire class $n$ function $f: X \rightarrow Z$ with $n \in \omega$, then there exists a Baire class 1 function $s: Y \rightarrow X$ so that $g \circ s=I d_{Y}$ and $f \circ s$ is Baire class $n$.

Corollary 7.2 For $f$ and $F$ defined in the previous diagram, if $f$ is Baire classe $n$ then $F$ is Baire class $n$ too.

Proof: Takes $X=A^{\omega}, Y=[0,1]$ and $Z=A^{\omega}$. By theorem 7.1 there exists a selector $s:[0,1] \rightarrow A^{\omega}$ so that $f \circ s$ is Baire class $n$. Then $F=\mu_{\theta} \circ f \circ s$ is Baire class $n$ too. 
Our aim is to extend the results of decidability to the function $F$.

C. Choffrut, H. Pelibossian and P. Simonnet [2] have shown that the continuity of the function $F$ is decidable with an algorithmic proof. We give a topological proof of this result and then show that we can also decide if $F$ is Baire class 1 .

Proposition 7.3 Let $F:[0,1] \rightarrow[0,1]$ so that there exist a function $f$ : $A^{\omega} \rightarrow A^{\omega}$ which verifies :

(1) Graph $(f)$ is definable in $S 1 S$.

(2) $\forall \alpha \in A^{\omega}: \mu_{\theta}(f(\alpha))=F\left(\mu_{\theta}(\alpha)\right)$.

Then we can decide if $F$ is continuous.

Proof: The function $\mathrm{F}$ is continuous iff its graph is closed. So let us prove that we can decide if $\operatorname{Graph}(F)$ is closed. Let $\boldsymbol{\mu}$ be defined by :

$$
\begin{aligned}
\boldsymbol{\mu}: A^{\omega} \times A^{\omega} & \rightarrow[0,1] \times[0,1] \\
(\alpha, \beta) & \mapsto\left(\mu_{\theta}(\alpha), \mu_{\theta}(\beta)\right)
\end{aligned}
$$

Note $H=\boldsymbol{\mu}^{-1}(\operatorname{Graph}(F))=\left\{(\alpha, \beta) \in A^{\omega} \times A^{\omega} \mid F\left(\mu_{\theta}(\alpha)\right)=\mu_{\theta}(\beta)\right\}=$ $\left\{(\alpha, \beta) \in A^{\omega} \times A^{\omega} \mid \mu_{\theta}(f(\alpha))=\mu_{\theta}(\beta)\right\}$. As $\theta$ is a Pisot number and $f$ definable in $S 1 S, H$ is definable in $S 1 S$.

If $\operatorname{Graph}(F)$ is closed, as $\boldsymbol{\mu}$ is continuous, $H$ is closed too. Conversely, if $H$ is closed, as $A^{\omega}$ is compact and $\boldsymbol{\mu}$ is continuous and surjective, $\operatorname{Graph}(F)=$ $\boldsymbol{\mu}\left(\boldsymbol{\mu}^{-1}(\operatorname{Graph}(F))\right)=\boldsymbol{\mu}(H)$ is compact. Then $F$ is continuous iff $H$ is closed. The set $H$ is recognizable by automaton so by proposition 5.3 we can decide if $F$ is continuous.

Proposition 7.4 Let $F:[0,1] \rightarrow[0,1]$ such that there exists a function $f:$ $A^{\omega} \rightarrow A^{\omega}$ which verifies :

(1) Graph $(f)$ is definable in $S 1 S$.

(2) $\forall \alpha \in A^{\omega}: \mu_{\theta}(f(\alpha))=F\left(\mu_{\theta}((\alpha))\right.$.

Then we can decide if $F$ is Baire class one.

Proof: For the proof we use an old result of W. Sierpinski :

a function $F: \mathbb{R} \longrightarrow \mathbb{R}$ is Baire class 1 iff its overgraph and its undergraph are $F_{\sigma}[24]$.

Let $H=\boldsymbol{\mu}^{-1}(G \uparrow(F))=\left\{(\alpha, \beta) \in A^{\omega} \times A^{\omega} \mid F\left(\mu_{\theta}(\alpha)\right)<\mu_{\theta}(\beta)\right\}$ we have $H=\left\{(\alpha, \beta) \in A^{\omega} \times A^{\omega} \mid \mu_{\theta}(f(\alpha))<\mu_{\theta}(\beta)\right\}$. As $\theta$ is a Pisot number and $f$ definable in $S 1 S, H$ is definable in $S 1 S$. 
By the same argument as in proposition 7.3 , it is easy to verify that $G \uparrow(F)$ is $F_{\sigma}$ iff $H$ is $F_{\sigma}$. To see this note that as $\boldsymbol{\mu}$ is surjective

$$
G \uparrow(F)=\boldsymbol{\mu}\left(\boldsymbol{\mu}^{-1}(G \uparrow(F))\right)=\boldsymbol{\mu}(H)
$$

As $\boldsymbol{\mu}$ is continuous if $G \uparrow(F)$ is $F_{\sigma}$ then $H$ is $F_{\sigma}$. Conversely if $H$ is $F_{\sigma}$, as $A^{\omega} \times A^{\omega}$ is compact, then $H$ is $K_{\sigma}$ (countable union of compact sets) and $G \uparrow(F)=\boldsymbol{\mu}(H)$ is $K_{\sigma}$ as a continuous image of a $K_{\sigma}$ set.

As $H$ is recognizable by automaton, by proposition 5.3 we can decide if $F$ is Baire class 1.

\section{Conclusion}

Let us talk about the asynchronuous case. An $\omega$-rational relation is a relation whose graph is recognized by a Büchi automaton, and for which transitions are labeled by couples of words $(u, v) \in A^{*} \times B^{*}$ instead of couples of letters $(a, b) \in A \times B$. They were first studied by F. Gire and M. Nivat, see $[9,6,7]$. Françoise Gire has shown that the problem of functionality is decidable for an $\omega$-rational relation. Recall that a set is analytic ( $\Sigma_{1}^{1}$ in the notation of Addison see [15]) if it is the continuous image of a Borel set. It is well known that Borel sets are analytics sets but that there exist analytics sets which are not Borel [15]. It is easy to see that $\omega$-rational relations are analytic sets. Recently O. Finkel has shown that there exist an $\omega$-rational relation which is not Borel [6]. From this he deduces many undecidability results [7]. It is easy to see that an $\omega$-rational function is of Baire class 2. Recently, C. Prieur $[20,21]$ has generalized the decidability of continuity to the $\omega$-rational functions. Moreover the overgraph (resp. undergraph) of an $\omega$-rational function is an $\omega$-rational relation. Unfortunately $O$. Finkel has shown the following theorem: the problem of knowing if an $\omega$-rational relation is $\Sigma_{i}^{0}\left(\operatorname{resp} . \boldsymbol{\Pi}_{i}^{0}\right)$ for $i=$ 1 and 2 is undecidable [7]. In addition, from O. Carton (personal communication) we have the following result: the problem of knowing if an $\omega$-rational function is totally discontinuous is undecidable. So we think that Baire class 1 is undecidable for the $\omega$-rational functions.

For ending we come back on finite word. The following theorem of Elgot Mezei [23] is well known: a rational relation which is a graph of a function $f$ with $f(\epsilon)=\epsilon$ is the composition of a left sequential function and a right sequential function. On infinite words the left part gives continuity, but the right part gives function of Baire class 2. We think that there exists a right subsequential function such that its on-the-fly extension on infinite words is not Baire class 1. Can we interpret points of continuity, as points that need only one register in an on the fly algorithm ? Finally note that the Booth canonical recoding 
is an $\omega$-rational relation with bounded delay, and all $\omega$-rational relation with bounded delay can be synchronized [9], this is what we have done.

The author would like to thank Serge Grigorieff for very hepful comments on a preliminary version of this paper. We thank also Pierre Delfini and Alain Hertzog for historical remarks. We would like to thank also the anonymous referees for suggestions which greatly improved the manuscript.

\section{References}

[1] J. R. Büchi. On a decision method in restricted second order arithmetic. Methodology and Philosophy of Science. Stanford Univ. Press, Calif. 1962. $1-11$.

[2] C. Choffrut, H. Pelibossian and P. Simonnet. Decision issues on functions realized by finite automata. Journal of Automata, Languages and Combinatorics 4(3). 1999. 171-182.

[3] A. Edgar. Classics On Fractals. Studies in non linearity, Westview Press. 2004.

[4] S. Eilenberg. Automata, Languages and Machines Vol A. Academic Press, New York London. 1974.

[5] M. D. Ercegovac, T. Lang. On-the-fly convertion of redundant into conventional representations. IEEE Trans. on Computers 36. 1987. 895-897.

[6] O. Finkel On the topological complexity of infinitary rational relations. Theor. Inform. Appl. 2003. 105-113.

[7] O. Finkel Undecidability of topological and arithmetical properties of infinitary rational relations. Theor. Inform. Appl. 2003. 115-126.

[8] G. Flory Topologie, Analyse. Vuibert. 1976. 130.

[9] C. Frougny, J . Sakarovitch. Synchronized relations of finite and infinite words. Theoret. Comput. Sci. 1993. 45-82.

[10] C. Frougny, B. Solomyak. On representation of integers in linear numeration systems. Ergodic Theory of $Z^{d}$-Actions. New Brunswick, New Jersey. 1996. $345-368$.

[11] C. Frougny. On-the-Fly algorithms and sequential machines. IEEE Trans. on Computers 49. 2000. 859-863.

[12] C. Frougny. Numeration Systems. Chapter 7 of M. Lothaire, Algebraic Combinatorics on Words. Cambridge University Press, 2002.

[13] F. Gire. Two decidability problems for infinite words. Inform. Proc. Letters 22. 1986. $135-140$. 
[14] L. H. Landweber. Decision problem for $\omega$-automata. Math. Systems. Theory 3. 1969. $376-384$.

[15] A. S. Kechris. Classical Descriptive Set Theory. Springer-Verlag. 1995.

[16] P. Kornerup. Digit-set convertions : Generalizations and applications. IEEE Trans. on Computers 43. 1994. 622-629.

[17] B. Maurey, J.P. Tacchi. Ludwig Scheeffer et les extensions du Théorème des Accroissements Finis. séminaire du Luxembourg, Travaux mathématiques, fascicule XIII. 2002. 1-60.

[18] J. M. Muller. Arithmétique des ordinateurs. Masson. Paris. 1989.

[19] D. Perrin J.-E. Pin. Infinite words, Automata, Semigroups, Logic and Games. volume 141 in the Pure and Applied Mathematics Series, Academic Press, Elsevier. 2004.

[20] C. Prieur. How to decide continuity of rationnal functions on infinite words. Theoret. Comput. Sci. 250. 2001. 71-82.

[21] C. Prieur. How to decide continuity of rationnal functions on infinite words (Errata). Theoret. Comput. Sci. 276. 2002. 445-447.

[22] J. Saint Raymond. Fonctions boréliennes sur un quotient. Bull. Soc. Math. France (2). 1976. 141-147.

[23] J. Sakarovitch Eléments de théorie des automates. Vuibert informatique. 2003.

[24] W. Sierpinski. Sur les fonctions de première classe. C. R. Acad. Sci. Paris 170. 1920. 919-922. 